\title{
Uncommon EGFR mutations in lung adenocarcinoma: features and response to tyrosine kinase inhibitors
}

\author{
Aurélien Brindel $^{1,2 \#}$, Wajd Althakfi, ${ }^{1,3,4 \#}$, Marc Barritault ${ }^{1,5}$, Emmanuel Watkin ${ }^{6}$, Jean-Michel Maury ${ }^{5,6,7}$, \\ Pierre-Paul Bringuier ${ }^{1,5}$, Nicolas Girard ${ }^{5,8}$, Marie Brevet ${ }^{1,5}$ \\ ${ }^{1}$ Institut de Pathologie Multi-sites des Hospices Civils de Lyon - Site Est et Plateforme de Pathologie Moléculaire, Hospices Civils de Lyon, Bron, \\ France; ${ }^{2}$ Département de Pneumologie, Centre Hospitalier Régional Universitaire de Nancy, Nancy, France; ${ }^{3}$ Department of Pathology, King \\ Saud University, Riyadh, Kingdom of Saudi Arabia; ${ }^{4}$ Service d'anatomie et de Cytologie Pathologiques, Centre Hospitalier Universitaire d'Amiens, \\ Amiens, France; ${ }^{5}$ Université Claude Bernard Lyon 1, Lyon, France; ${ }^{6}$ Cypath, Villeurbanne, France; ${ }^{7}$ Département de Chirurgie Thoracique, \\ Transplantation Pulmonaire et Cardio-pulmonaire, Hôpital Cardio-vasculaire et Pneumologique de Louis Pradel, Hospices Civils de Lyon, Bron, \\ France; ${ }^{8}$ Institut du Thorax, Institut Curie, Paris, France \\ Contributions: (I) Conception and design: M Brevet, N Girard; (II) Administrative support: M Brevet, N Girard; (III) Provision of study materials or \\ patients: N Girard, JMM, PPB, M Barritault, M Brevet, E Watkin; (IV) Collection and assembly of data: W Althakfi, A Brindel; (V) Data analysis and \\ interpretation: A Brindel, W Althakfi, M Brevet, N Girard, M Barritault, PP Bringuier; (VI) Manuscript writing: All authors; (VII) Final approval of \\ manuscript: All authors. \\ "These authors contributed equally to this work. \\ Correspondence to: Marie Brevet. Institut de Pathologie Multi-sites des HCL-Site Est, Centre de biologie et de Pathologie est, Hospices Civils de \\ Lyon, 59 boulevard Pinel, 69677 Bron, France. Email: marie.brevet01@chu-lyon.fr.
}

Background: EGFR-mutant non-small cell lung cancer (NSCLC) is a heterogeneous disease. The treatment for frequent EGFR mutations relies on tyrosine kinase inhibitors (TKIs); the clinical and therapeutic significance of uncommon EGFR mutations is uncertain.

Methods: This is a single-center retrospective study of patients with EGFR-mutant lung cancer (2009-2017). Molecular analyses of EGFR exons 18-21 were performed. Only patients with uncommon mutations were included (p.Glu709X, p.Gly719X, p.Ala767_Val769 dup, p.Ser768Ile, and p.Leu861Gln).

Results: Among 6,747 tumor samples, 95 out 820 patients (11.6\%) harbored 113 uncommon EGFR mutations. There were 50 metastatic NSCLC patients for whom the median OS was 18.0 months (95\% CI: 15, 32). In this population, the p.Leu861Gln uncommon exon 21 EGFR mutation was associated with poor prognosis (HR: 2.96, 95\% CI: 1.39, 6.31; P=0.003). Among those harboring a single uncommon EGFR mutation, median OS was 27.6 months (95\% CI: 10.8, not attained) in patients who were treated by chemotherapy only (n=13) versus 6.0 months (95\% CI: 2.4 , not attained) in patients exclusively treated with a first or second-EGFR-TKI (n=9; HR: 0.27, 95\% CI: 0.09, 0.78; P=0.01. In patients with a single uncommon EGFR mutation, first-line chemotherapy was associated with a better overall survival than TKIs (HR: 0.31, 95\% CI: 0.15, 0.68; $\mathrm{P}=0.002)$. In patients who received first or second-EGFR-TKI as first-line treatment $(\mathrm{n}=26)$, OS was significantly better for those with two uncommon EGFR mutations than those with a single uncommon mutation (HR: 0.07, 95\% CI: 0.009, 0.54; P=0.001).

Conclusions: In conclusion, uncommon EGFR mutations may be associated with a poor outcome and the data challenge the use of first-generation TKI in such patients, however first-line TKI is more effective in cases of double uncommon mutations and such patients should be treated accordingly.

Keywords: Lung cancer; adenocarcinoma; uncommon EGFR mutation; p.Leu861Gln

Submitted Dec 26, 2019. Accepted for publication Jun 02, 2020.

doi: $10.21037 /$ jtd-19-3790

View this article at: http://dx.doi.org/10.21037/jtd-19-3790

(c) Journal of Thoracic Disease. All rights reserved. 


\section{Introduction}

Lung cancer is the first cause of cancer-related death, and it is of note that the majority of patients are diagnosed with metastatic disease (1). Non-small-cell lung cancer (NSCLC), and, more specifically adenocarcinoma, represents the most frequent histological type. Epidermal growth factor receptor $(E G F R)$ mutation is found in approximately $11 \%$ of Caucasian patients with advanced metastatic lung adenocarcinoma (2), and such mutations predict the efficacy of EGFR tyrosine kinase inhibitors (TKIs) that are the standard-of-care first-line treatment in such patients (3-5). EGFR mutations are, however, heterogeneous; the most common are exon 19 deletions and p.Leu858Arg point mutation are each observed in $40-45 \%$ of cases (6), but several other mutations, so-called uncommon EGFR mutations, are spread within exons 18-21 (6).

Whether available EGFR TKIs are effective in patients with uncommon $E G F R$ mutations is currently unclear as response to treatment is variable (7). For instance, while p.Glu719X and p.Ser768Gln have been reported to be associated with response to second generation TKIs such as afatinib in the landmark trials of this inhibitor (6), other mutations may predict primary resistance or lower sensitivity to first- and second-generation EGFRTKIs, such as the p.Thr790Met mutation in exon 20 and p.Leu861Gln mutation in exon 21, respectively (8-11). However, most of the data are from retrospective, post hoc studies with small and heterogeneous groups (12).

Herein we present the results of a large study of patients with lung adenocarcinoma genotyped for EGFR mutations; the data describe the frequency and the prognosis of uncommon EGFR mutations.

\section{Methods}

This study was carried out retrospectively using the database of the pathology department of the Hospices Civils de Lyon, Lyon, France, that prospectively collected results of EGFR genotyping for all consecutive patients with lung adenocarcinoma referred for molecular biology testing from 2009 to 2017. The trial was conducted in accordance with the Declaration of Helsinki and the Harmonized Tripartite Guideline for Good Clinical Practice from the International Conference on Harmonization. Patient were informed about this research project on their data and the present study was approved by the institutional ethics committee.

\section{EGFR mutational analyses}

EGFR mutation genotyping was conducted as follows: after laser microdissection, DNA was extracted using the QIAMP DNA micro kit (QIAGEN, Venlo, Netherlands) according to the manufacturer's instructions. From 2009 to March 2016, mutation status within EGFR gene exons 18, 19, 20, and 21 were detected using a polymerase chain reaction (PCR) and Sanger sequencing. After March 2016, exons 18 to 21 of EGFR were sequenced by Next Generation Sequencing (NGS) using a custom panel and Ion Personal Genome Machine (PGM Ion Torrent, ThermoFisher Scientific, Waltham, MA, USA). Mutations were validated if present in two independent replicates.

\section{Identification of uncommon EGFR mutations}

This analysis included all cases, irrespective of clinical stage or specimen origin. Specimens with non-interpretable results or absence of EGFR mutations were excluded. Duplicates (multiple samples for the same patient) were identified and the most relevant analysis was included; the others were excluded. Within each of the four analyzed exons, and using Alamut ${ }^{\circledR}$ Visual software (version 2.11, Interactive Biosoftware, Rouen, France), mutations involving the same amino acids were identified in order to create several molecular subgroups according to the location of the mutations.

$E G F R$ mutations were separated according to the known associated clinical issues. EGFR exon 19 deletion and p.Leu858Arg mutation were excluded because of known sensitivity to TKI. p.Thr790Met and exon 20 insertions were excluded because these are known to be associated with resistance to first- and second-generation EGFR TKIs. All other mutations were considered as "uncommon" EGFR mutations.

\section{Clinical data}

The study population included only patients harboring an uncommon EGFR mutation. For these, clinical data was collected using a questionnaire that was sent to the oncologist in charge of the patient. This study was conducted in accordance with Good Clinical Practices and performed after formal agreement by the ethics committee of the Hospices Civils de Lyon. The questionnaire collected data regarding the date of diagnosis, date of death, smoking 
status, clinical stage, presence of distant metastases, and treatment modalities (surgery, chemotherapy and TKI). Overall survival (OS) was calculated from the time from the date of diagnosis to death or the date of last followup. TNM stage was assessed according to the eighth International Association for the Study of Lung cancer (IASLC) Staging Project (13).

\section{Statistical methods}

Descriptive statistics were carried out to estimate the frequency of uncommon mutations. EGFR mutations was described by mutation location (exon) and type (nomenclature of involved amino acid) and expressed as a percentage. In the studied population, comparison of pathological characteristics between different molecular subgroups was analyzed using the independent t-test for age, and Chi-squared test for sex and smoking status. The Kaplan-Meier method was used for survival analyses. Groups were compared in univariate analysis using the Log-rank test. Multivariate analyses were conducted using a Cox model including the different types of mutation, sex, age, and TNM stage. Hazard ratios (HRs) and their $95 \%$ confidence intervals (CIs) were computed. A P value of $<0.05$ was considered significant, and statistical analyses were performed using RStudio Version 1.1.456, RStudio (Boston, MA, USA).

\section{Results}

\section{Frequency of uncommon EGFR mutations}

From 2009 to May 2017 a total of 7,539 analyses were performed for EGFR genotyping at our center and among these an EGFR mutation was identified in 1,121 (14.9\%). After exclusion of duplicates (more than one sample per patient), a total 6,747 patients were tested for EGFR mutation and 820 patients were diagnosed with EGFRmutant adenocarcinoma (12.2\%). Among them, 725 patients $(88.4 \%)$ had a tumor that harbored known significant mutations (including p.Leu858Arg in exon 21, Del exon 19, Ins exon 20 and p.Thr790Met in exon 20). Thus, 95 (11.6\%) patients had a tumor that harbored at least one uncommon EGFR mutation, and were included in the analysis. Overall, 68 cases $(71.6 \%)$ had a single uncommon EGFR mutation, 18 cases $(18.9 \%)$ a double uncommon EGFR mutation; nine patients $(9.5 \%)$ had both one uncommon EGFR mutation and one frequent p.Leu858Arg mutation. The 113 uncommon EGFR mutations were the following: insertions of exon 18 [p.Glu709X (16/113, 14.2\%) and p.Gly719X $(40 / 113,35.4 \%)]$, duplications and substitutions of exon 20 [p.Ala767_Val769 dup (17/113, 15.0\%), p.Ser768Ile $(18 / 113,15.9 \%)$ ] and substitutions of exon 21 [p.Leu861Gln (22/113, 19.5\%)]. The distribution of EGFR uncommon mutations among the 95 patients is presented in Figure 1; 68 patients harbored a single EGFR uncommon mutations, and 27 patients harbored EGFR co-mutations.

\section{Clinical characteristics and treatment}

The median age of patients with a tumor harboring an uncommon EGFR mutation was 68.3 years (range, 39 to 89 years), $57.8 \%$ of patients $(55 / 95)$ were women and, among those with data, $51.4 \%(36 / 70)$ were current or former smokers (Table 1). Clinical data and follow-up were available for 73 patients (76.8\%). Overall, 17 (17.9\%) patients underwent surgical resection, including three patients with p.Leu858Arg co-mutation. Among these, nine patients subsequently received systemic treatment including chemotherapy (eight patients), EGFR TKI (three patients), or both (two patients). Eight patients did not receive any subsequent systemic treatment.

Among the 56 patients with metastatic NSCLC (patients diagnosed with metastasis at diagnosis and who did not received any surgical treatment), 51 received a systemic treatment, including at least one line of chemotherapy for 40 patients, at least one first or second-EGFR-TKI for 38 patients; 27 patients received both chemotherapy and TKI. Five patients received neither chemotherapy nor TKI due to rapid death after diagnosis (four patients) or poor performance status (one patient). First-line treatment was chemotherapy for 24/51 (47.1\%) patients, erlotinib for 10/51 (19.6\%) patients, gefitinib for 14/51 (27.5\%) patients, and afatinib for three (5.9\%) patients (Figure S1; Table S1); 14/51 (27.5\%) patients with metastatic disease had no second line due to premature death or a poor performance status (Figure S2).

\section{Overall survival according to mutation}

Survival analyses were performed after exclusion of the six patients with double mutations including p.Leu858Arg. Median OS was 27.6 months (95\% CI: 18.0, 33.6) for the study population ( $\mathrm{n}=67$, Figure 2), and 18.0 months ( $95 \%$ CI: $14.4,32.4)$ for patients with metastatic NSCLC ( $=50$, Figure 3). Among patients with metastatic NSCLC and 


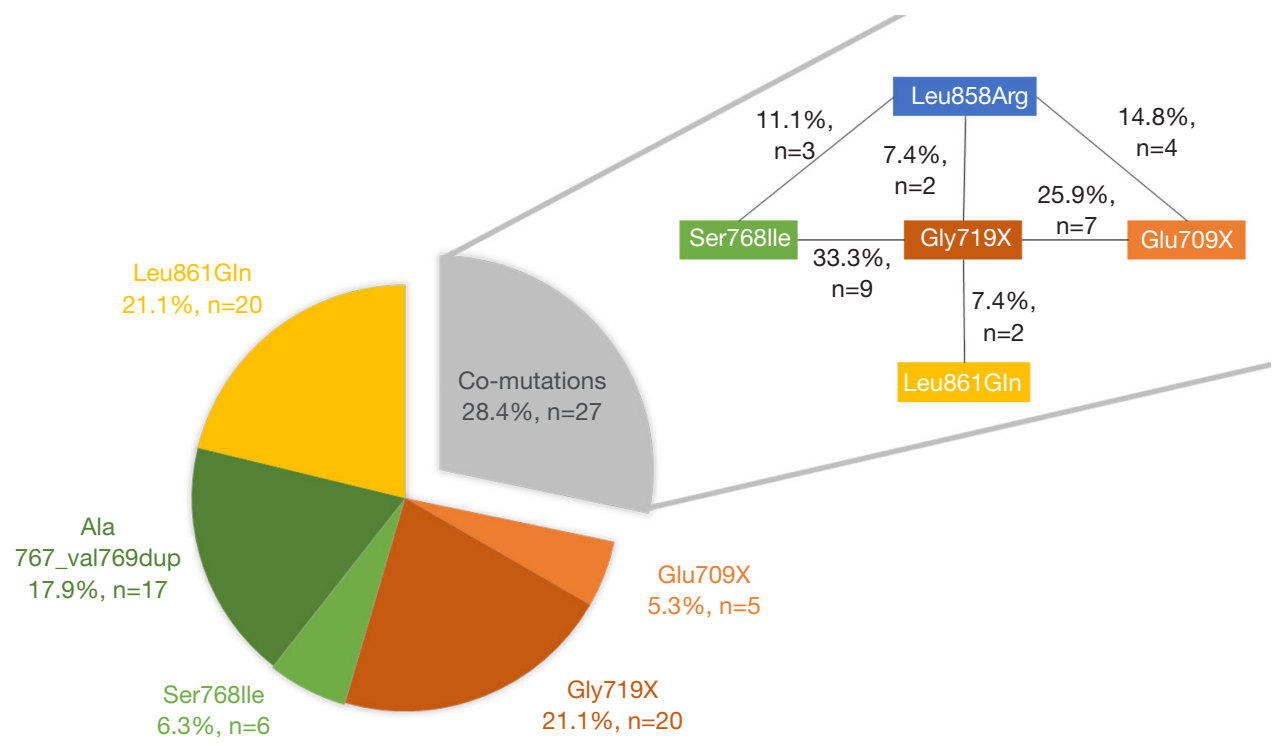

Figure 1 Frequency of uncommon EGFR mutations. Distribution of EGFR uncommon mutations among the 95 patients (left) and the details of the 27 co-mutated samples (right).

a single uncommon EGFR mutation ( $\mathrm{n}=43$ ), median OS according to the $E G F R$ uncommon mutation did not show any significance (Figures S3). Nevertheless, as compared to all other uncommon mutations, exon 21 (p.Leu861Gln) was significantly associated with poorer OS (HR: $2.96,95 \%$ CI: $1.39,6.31 ; \mathrm{P}=0.003)$. No other clinical factor as TNM staging, sex, smoking status, age over 75 were significant in monovariate analysis (data not shown).

\section{Overall survival according to treatment}

Among patients with metastatic NSCLC harboring a single EGFR uncommon mutation (Table S2), median OS was 27.6 months (95\% CI: 10.8, not attained) in patients who were treated by chemotherapy only $(\mathrm{n}=13)$ versus 6.0 months (95\% CI: 2.4, not attained) in patients exclusively treated with a first or second-EGFR-TKI (n=9; HR: 0.27, 95\% CI: 0.09, 0.78; P=0.01, Figure 4). The significance was still observed when we compared patients exclusively treated with a TKI $(n=9)$ versus patients who had received chemotherapy and TKI ( $\mathrm{n}=21$; HR: $0.29,95 \%$ CI: 0.12 , $0.71 ; \mathrm{P}=0.004$; Figure $S 4 A$ ) or versus all other patients (chemotherapy +/- TKI, n=34; HR: 0.26, 95\% CI: 0.11, $0.60 ; \mathrm{P}=0.0008$; Figure $S 4 B)$.

Considering only first-line treatments in patients with metastatic NSCLC harboring a single EGFR uncommon mutation, chemotherapy was associated with a more prolonged OS (HR: 0.31, 95\% CI: 0.15, 0.68; $\mathrm{P}=0.002$ ); the median OS was 27.6 months (95\% CI: $10.8,49.2)$ for chemotherapy versus 11.4 months (95\% CI: 6.0, 18.0) for TKI (Figure 5). Median OS according to first-line treatment and type of uncommon EGFR mutation did not show any significant result (Figure S5).

In patients who received EGFR TKI as first-line treatment $(n=26)$, OS was significantly better for patients with a tumor harboring two uncommon EGFR mutations (Table S3) than for patients with a tumor harboring a single uncommon EGFR mutation (HR: 0.07, 95\% CI: 0.009, 0.54; $\mathrm{P}=0.001)$.

\section{Discussion}

Through the analysis of a large cohort of patients with molecularly-characterized lung adenocarcinomas, the present study shows the variety of therapeutic strategies employed in a real-life setting, and the poor outcomes of the patients harboring uncommon EGFR mutation.

The frequency of uncommon mutations herein was similar to that reported in previous studies in Caucasian patients; in the Biomarqueurs France study, which included 10,117 patients, uncommon EGFR mutations accounted for approximately $10 \%$ of all EGFR-mutated cases (2). However, in an Asian cohort of 1261 patients, Wu et al. reported a higher rate (12.4\%) of uncommon 
Table 1 Characteristics of patients harboring uncommon EGFR mutation(s)

\begin{tabular}{|c|c|}
\hline & Total population $(n=95)$ \\
\hline \multicolumn{2}{|l|}{ Sex, n (\%) } \\
\hline Female & $55(57.9)$ \\
\hline Male & $40(42.1)$ \\
\hline Median age, years (range) & $68.3(39-89)$ \\
\hline \multicolumn{2}{|l|}{ Smoking history, n (\%) } \\
\hline Never & $34(35.8)$ \\
\hline Current or former & $36(37.9)$ \\
\hline Missing data & $25(26.3)$ \\
\hline \multicolumn{2}{|l|}{ Stage, n (\%) } \\
\hline 1 & $6(6.3)$ \\
\hline II & $9(9.5)$ \\
\hline III & $8(8.4)$ \\
\hline IV & $50(52.6)$ \\
\hline Missing data & $22(23.2)$ \\
\hline Treatment, n (\%) & $73(76.8)$ \\
\hline $\begin{array}{l}\text { Surgical treatment } \pm \text { systemic } \\
\text { therapy }\end{array}$ & $17^{\mathrm{a}}(17.9)$ \\
\hline $\begin{array}{l}\text { Targeted treatment and } \\
\text { chemotherapy }\end{array}$ & $2^{b}(11.8)^{c}$ \\
\hline Chemotherapy only & $6^{b}(35.3)^{c}$ \\
\hline Targeted treatment only & $1(5.9)^{\mathrm{c}}$ \\
\hline Surgery only & $8^{b}(47.1)^{c}$ \\
\hline Systemic treatment & $56^{a}(58.9)$ \\
\hline $\begin{array}{l}\text { Targeted treatment and } \\
\text { chemotherapy }\end{array}$ & $27(48.2)^{c}$ \\
\hline Chemotherapy only & $13(23.2)^{\mathrm{c}}$ \\
\hline Targeted treatment only & $11^{b}(19.6)^{c}$ \\
\hline None & $5^{\mathrm{d}}(8.9)^{\mathrm{c}}$ \\
\hline Missing data & $22^{\mathrm{a}}(23.2)$ \\
\hline
\end{tabular}

ancluding three patients with p.Leu858Arg co-mutation; ${ }^{b}$ including one patient with p.Leu858Arg co-mutation; ' $a m o n g$ those concerned; ${ }^{d}$ including two patients patients with p.Leu858Arg co-mutation.

EGFR mutations (14), while Li et al. reported about 5\% uncommon $E G F R$ mutation among 2,043 patients with $E G F R$ mutations (15). Nevertheless, the frequency of uncommon mutations is completely dependent of the

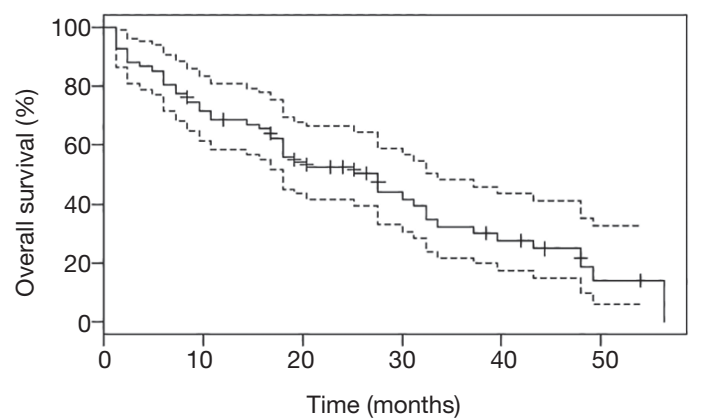

Figure 2 Overall survival (OS) for the study population of patients with uncommon EGFR mutation, after exclusion of those with p.Leu858Arg co-mutations. Point estimate (continuous line) and 95\% CI (discontinuous line) are shown.

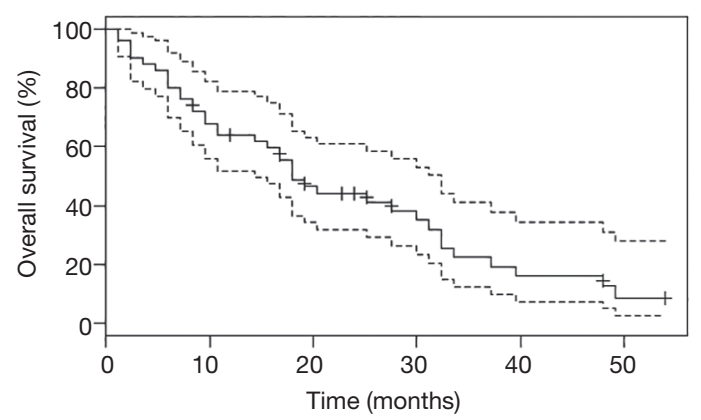

Figure 3 Overall survival (OS) for patients with metastatic NSCLC with uncommon EGFR mutation, after exclusion of those with p.Leu858Arg co-mutation. Point estimate (continuous line) and $95 \% \mathrm{CI}$ (discontinuous line) are shown.

technique used in routine diagnosis and therefore limits any interpretation of such data. More interestingly, a higher proportion of current or former smokers was found herein than in Asian EGFR-mutated populations, highlighting the need for systematic genotyping irrespective of patient smoking status $(15,16)$.

A striking finding of the present study is that the choice of treatment for each mutation was variable that reflects the absence of recommendation or consensus for uncommon EGFR mutations. This may be explained by the almost complete lack of evidence for the use of TKIs available during the study period. First-line treatment was chemotherapy for almost half of the metastatic patients; EGFR TKI was the preferred treatment for exon 18 and 21 mutations while most exon 20 mutations received chemotherapy.

The median OS in metastatic patients herein was similar 


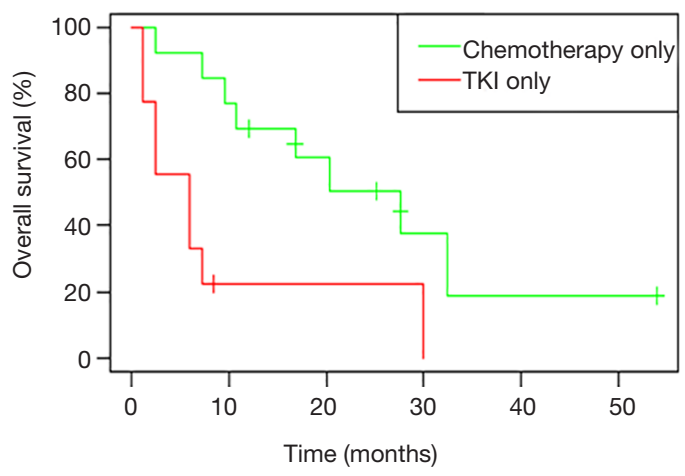

Figure 4 Overall survival (OS) for patients with a single uncommon EGFR mutation according to treatment, all lines $(n=22)$. Patients treated by chemotherapy and TKI were excluded $(n=21)$.

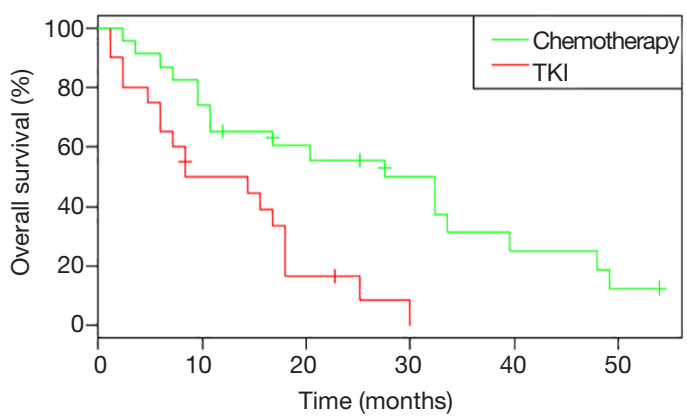

Figure 5 Overall survival (OS) for patients with a single uncommon EGFR mutation according to first-line treatment received $(n=43)$.

to that was previously reported for uncommon $E G F R$ mutations in clinical trials: in the pooled analysis of the LUX-LUNG 2, 3, and 6 trials in which 38 patients (with tumor harboring uncommon mutations including point mutations or duplications in exons 18-21) were treated by afatinib, the median OS was about 13 months when patients were treated with first-generation TKIs $(17,18)$ and 19.4 months (95\% CI: 16.4, 26.9) (6). An important result of this study is that p.Leu861Gln mutations were associated with poor prognosis compared to other uncommon mutations; this is in line with the data reported by Leduc et al. in another cohort of Caucasian patients as most patients with p.Leu861Gln were treated with chemotherapy first and had better progression-free survival (PFS) than patients treated with TKI (8). This is supported by in vitro studies on mutant cell lines that found that p.Leu861Gln mutations enhanced kinase activity via a strong activation of Stat5 but, surprisingly, they seem not increase drug sensitivity to first generation TKI in clinical use (19). In parallel, first-line treatment seems to be a significant predictor of outcome because more than a quarter of patients did not have access to second-line of treatment in the present study, highlighting the potential aggressiveness associated with uncommon EGFR mutations (3). Taken together, these results suggest that chemotherapy should be the first-line treatment in the presence of a p.Leu861Gln mutation.

Another interesting finding of the present study is that patients with double uncommon EGFR mutations receiving any TKI as first-line treatment had better survival compared to patients with single mutations, independently of their association with a p.Leu858Arg mutation. Pilotto et al. reported a trend towards better OS in double EGFR mutated patients; the median OS was 21.3 (95\% CI: 0.69, not attained) months in this population compared to 14.5 (95\% CI: 8.1, 22.5) for all uncommon mutations (17). Nevertheless, in the latter study, all double mutations included at least one mutation known for sensitivity to firstor second-generation of TKI as p. Leu858Arg or Exon 19 deletions and therefore is not comparable to that reported herein. Kimura et al. did, however, report a case of double uncommon mutation (p.Gly719X and p.Leu861Gln), and found increased sensitivity to afatinib (20), which is confirmed here in a larger population that included other targeted therapies. It is of note that Zou et al. predicted sensitivity to gefitinib/erlotinib for $E G F R$ mutations in NSCLC based on structural interaction fingerprints and multilinear principal component analysis (21); it would therefore be of great interest to study the conformation of the EGFR protein in case of double uncommon mutation in order to explain the gain in TKI sensitivity. More generally, as the presence of a double mutation (be this double uncommon, or uncommon/common) could change TKI sensitivity, we believe that a EGFR sequencing approach is probably better than targeted molecular EGFR analyses as the former are likely to detect such combined mutations.

The present study is one of the largest studies investigating frequency, clinical characteristics, and treatment outcomes of patients with uncommon EGFR mutations. However, it does have some intrinsic limitations related to its retrospective nature; treatments were not distributed in a randomized manner; we were unable to reliably collect PFS data as well as some clinical data such as clinical and imaging features of TKI resistance; 
moreover, the study started in 2009 and only a few patients received second- or third-generation EGFR TKIs while evidence now exists regarding a potential effectiveness of such inhibitors in case of uncommon EGFR mutations in the first-line and beyond (6,20,22-25). This work should therefore be updated at a later date to take into account these new drugs.

In conclusion, uncommon EGFR mutations may be associated with a poor outcome and the data challenge the use of first-generation TKI in such patients, however firstline TKI is more effective in cases of double uncommon mutations and such patients should be treated accordingly.

\section{Acknowledgments}

The authors thank all the pathologists who sent samples to the molecular diagnosis platform of the Hospices Civils de Lyon, and the clinicians who gave clinical data and contributed to this work. They also thank the technical team for molecular analysis, Dr Philip Robinson (DRCI, Hospices Civils de Lyon) for help in manuscript preparation, and the Biological Resource Centre of the Hospices Civils de Lyon (Tissu-tumorothèque Est).

Funding: This work was supported by Boehringer Ingelheim by unrestricted grant and by the French national cancer Institute (INCa) through the funding of routine genotyping of EGFR mutations in patients with NSCLC.

\section{Footnote}

Data Sharing Statement: Available at http://dx.doi. org/10.21037/jtd-19-3790

Conflicts of Interest: All authors have completed the ICMJE uniform disclosure form (available at http://dx.doi. org/10.21037/jtd-19-3790). AB reports grants from Boerhinger Ingelheim, during the conduct of the study; MB reports grants from Boerhinger Ingelheim, from null, outside the submitted work. The other authors have no conflicts of interest to declare.

Ethical Statement: The authors are accountable for all aspects of the work in ensuring that questions related to the accuracy or integrity of any part of the work are appropriately investigated and resolved. The trial was conducted in accordance with the Declaration of Helsinki and the Harmonized Tripartite Guideline for Good Clinical Practice from the International Conference on
Harmonization. This study was reviewed and approved by the Research Ethics Board of the Hospices civils de Lyon (approval number 17-151) and the Hoffmann-La Roche global review committee. All patients enrolled completed the informed consent form.

Open Access Statement: This is an Open Access article distributed in accordance with the Creative Commons Attribution-NonCommercial-NoDerivs 4.0 International License (CC BY-NC-ND 4.0), which permits the noncommercial replication and distribution of the article with the strict proviso that no changes or edits are made and the original work is properly cited (including links to both the formal publication through the relevant DOI and the license). See: https://creativecommons.org/licenses/by-nc-nd/4.0/.

\section{References}

1. Reck M, Rabe KF. Advanced Non-Small-Cell Lung Cancer. N Engl J Med 2017;377:1999.

2. Barlesi F, Mazieres J, Merlio JP, et al. Routine molecular profiling of patients with advanced non-small-cell lung cancer: Results of a 1-year nationwide programme of the French Cooperative Thoracic Intergroup (IFCT). Lancet 2016;387:1415-26.

3. Siegelin MD, Borczuk AC. Epidermal growth factor receptor mutations in lung adenocarcinoma. Lab Invest 2014;94:129-37.

4. Bean J, Riely GJ, Balak M, et al. Acquired resistance to epidermal growth factor receptor kinase inhibitors associated with a novel T854A mutation in a patient with EGFR-mutant lung adenocarcinoma. Clin Cancer Res 2008;14:7519-25.

5. Kosaka T, Yatabe Y, Endoh H, et al. Analysis of epidermal growth factor receptor gene mutation in patients with nonsmall cell lung cancer and acquired resistance to gefitinib. Clin Cancer Res 2006;12:5764-9.

6. Yang JCH, Sequist LV, Geater SL, et al. Clinical activity of afatinib in patients with advanced non-small-cell lung cancer harbouring uncommon EGFR mutations: A combined post-hoc analysis of LUX-Lung 2, LUX-Lung 3, and LUX-Lung 6. Lancet Oncol 2015;16:830-8.

7. Beau-Faller M, Prim N, Ruppert AM, et al. Rare EGFR exon 18 and exon 20 mutations in non-small-cell lung cancer on 10117 patients: A multicentre observational study by the French ERMETIC-IFCT network. Ann Oncol 2014;25:126-31.

8. Leduc C, Merlio JP, Besse B, et al. Clinical and molecular 
characteristics of non-small-cell lung cancer (NSCLC) harboring EGFR mutation: results of the nationwide French Cooperative Thoracic Intergroup (IFCT) program. Ann Oncol 2017;28:2715-24.

9. Yasuda H, Kobayashi S, Costa DB. EGFR exon 20 insertion mutations in non-small-cell lung cancer: Preclinical data and clinical implications. Lancet Oncol 2012;13:e23-31.

10. Wu JY, Wu SG, Yang CH, et al. Lung cancer with epidermal growth factor receptor exon 20 mutations is associated with poor gefitinib treatment response. Clin Cancer Res 2008;14:4877.

11. Woo HS, Ahn HK, Lee HY, et al. Epidermal growth factor receptor (EGFR) exon 20 mutations in non-smallcell lung cancer and resistance to EGFR-tyrosine kinase inhibitors. Invest New Drugs 2014;32:1311-5.

12. Mitsudomi T, Yatabe Y. Mutations of the epidermal growth factor receptor gene and related genes as determinants of epidermal growth factor receptor tyrosine kinase inhibitors sensitivity in lung cancer. Cancer Sci 2007;98:1817-24.

13. Goldstraw P, Chansky K, Crowley J, et al. The IASLC lung cancer staging project: Proposals for revision of the TNM stage groupings in the forthcoming (eighth) edition of the TNM Classification for lung cancer. J Thorac Oncol 2016;11:39-51.

14. Wu JY, Yu CJ, Chang YC, et al. Effectiveness of tyrosine kinase inhibitors on « uncommon » epidermal growth factor receptor mutations of unknown clinical significance in non-small cell lung cancer. Clin Cancer Res 2011;17:3812-21.

15. Li H, Wang C, Wang Z, et al. Efficacy and long-term survival of advanced lung adenocarcinoma patients with uncommon EGFR mutations treated with 1st generation EGFR-TKIs compared with chemotherapy as first-line therapy. Lung Cancer 2019;130:42-9.

16. Yang JCH, Wu YL, Schuler M, et al. Afatinib versus cisplatin-based chemotherapy for EGFR mutation-positive lung adenocarcinoma (LUX-Lung 3 and LUX-Lung 6): Analysis of overall survival data from two randomised, phase 3 trials. Lancet Oncol 2015;16:141-51.

Cite this article as: Brindel $\mathrm{A}$, Althakfi $\mathrm{W}$, Barritault $\mathrm{M}$, Watkin E, Maury JM, Bringuier PP, Girard N, Brevet M. Uncommon EGFR mutations in lung adenocarcinoma: features and response to tyrosine kinase inhibitors. J Thorac Dis 2020;12(9):4643-4650. doi: 10.21037/jtd-19-3790
17. Pilotto S, Rossi A, Vavalà T, et al. Outcomes of FirstGeneration EGFR-TKIs Against Non-Small-Cell Lung Cancer Harboring Uncommon EGFR Mutations: A Post Hoc Analysis of the BE-POSITIVE Study. Clin Lung Cancer 2018;19:93-104.

18. Watanabe S, Minegishi Y, Yoshizawa H, et al. Effectiveness of gefitinib against non-small-cell lung cancer with the uncommon EGFR mutations G719X and L861Q. J Thorac Oncol 2014;9:189-94.

19. Massarelli E, Johnson FM, Erickson HS, et al. Uncommon Epidermal Growth Factor Receptor mutations in nonsmall cell lung cancer and their mechanisms of EGFR tyrosine kinase inhibitors sensitivity and resistance. Lung Cancer 2013;80:235-41.

20. Kimura S, Tanaka K, Harada T, et al. Sensitivity of epidermal growth factor receptor with single or double uncommon mutations to afatinib confirmed by a visual assay. Cancer Sci 2018;109:3657-61.

21. Zou B, Lee VHF, Yan H. Prediction of sensitivity to gefitinib/erlotinib for EGFR mutations in NSCLC based on structural interaction fingerprints and multilinear principal component analysis. BMC Bioinformatics 2018;19:88.

22. Kobayashi Y, Mitsudomi T. Not all epidermal growth factor receptor mutations in lung cancer are created equal: Perspectives for individualized treatment strategy. Cancer Sci 2016;107:1179-86.

23. Heigener DF, Schumann C, Sebastian M, et al. Afatinib in Non-Small Cell Lung Cancer Harboring Uncommon EGFR Mutations Pretreated With Reversible EGFR Inhibitors. Oncologist 2015;20:1167-74.

24. Leventakos K, Kipp BR, Rumilla KM, et al. S768I mutation in EGFR in patients with lung cancer. J Thorac Oncol 2016;11:1798-801.

25. Duan H, Peng Y, Cui H, et al. Effectiveness of afatinib after ineffectiveness of gefitinib in an advanced lung adenocarcinoma patient with a single EGFR exon 20 S768I mutation: A case report. Onco Targets Ther 2018;11:2303-9. 


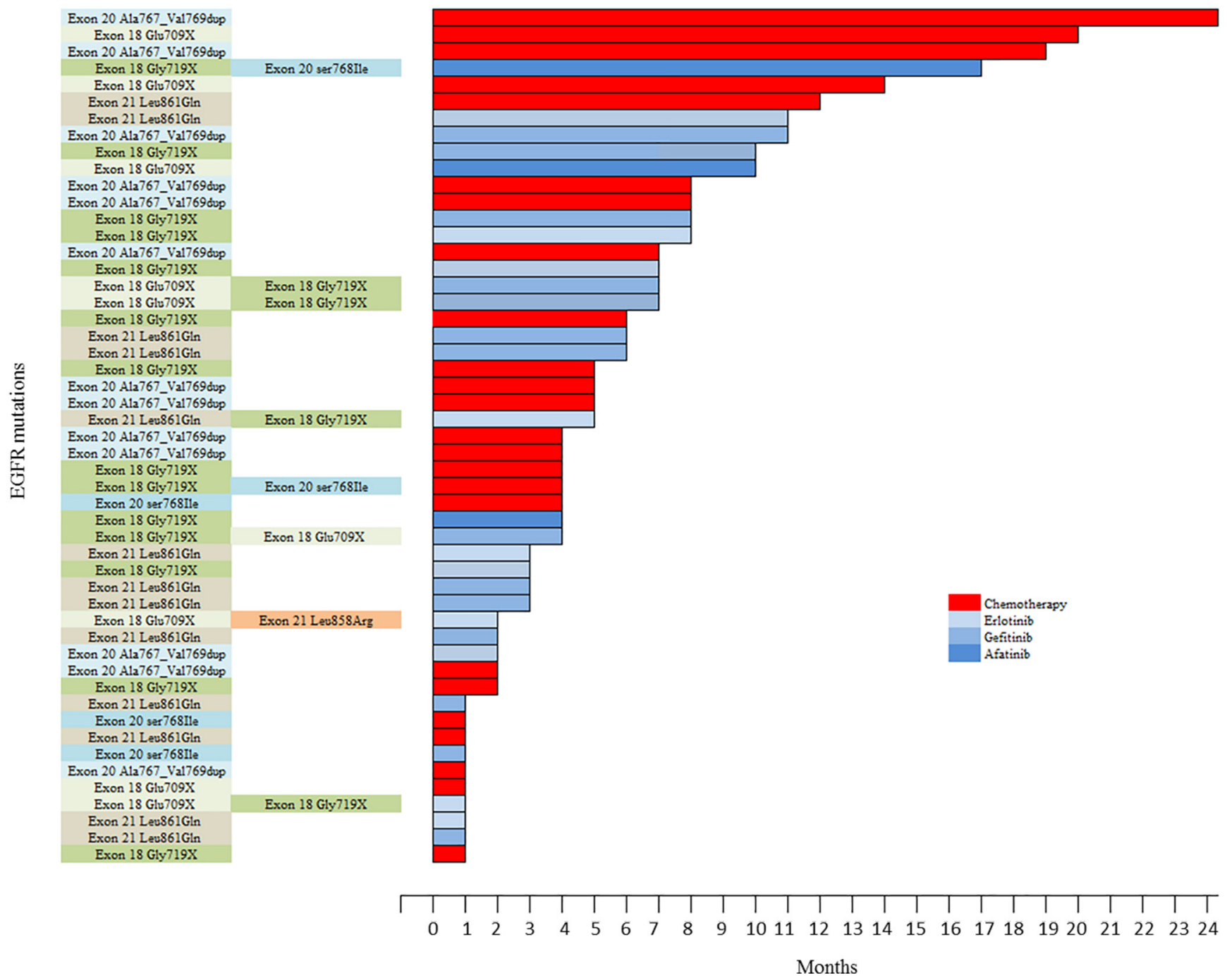

Figure S1 Swimmer plot considering the 51 metastatic patients who received first-line chemotherapy or tyrosine kinase inhibitor. Each unit correspond to one month of therapy. 
Table S1 Treatment details for the patients with metastatic NSCLC according to the line of treatment $(n=56)$

\begin{tabular}{|c|c|}
\hline Type to treatment & $\mathrm{N}$ \\
\hline Targeted therapy & 38 \\
\hline Erlotinib & 19 \\
\hline First-line & $10^{a}$ \\
\hline Second-line & 6 \\
\hline Third- line & 2 \\
\hline Other line & 1 \\
\hline Gefitinib & 18 \\
\hline First-line & 14 \\
\hline Second-line & 4 \\
\hline Other line & 0 \\
\hline Afatinib & 9 \\
\hline First-line & 3 \\
\hline Second-line & 2 \\
\hline Third-line & 3 \\
\hline Fourth-line & 1 \\
\hline Other line & 0 \\
\hline Chemotherapy & 40 \\
\hline First-line & 24 \\
\hline Second-line & 24 \\
\hline Third-line & 16 \\
\hline Fourth-line & 10 \\
\hline Other line & 7 \\
\hline None & $5^{\mathrm{b}}$ \\
\hline
\end{tabular}

including one patient with L858R co-mutation; 'including two patients with p.Leu858Arg co-mutation.

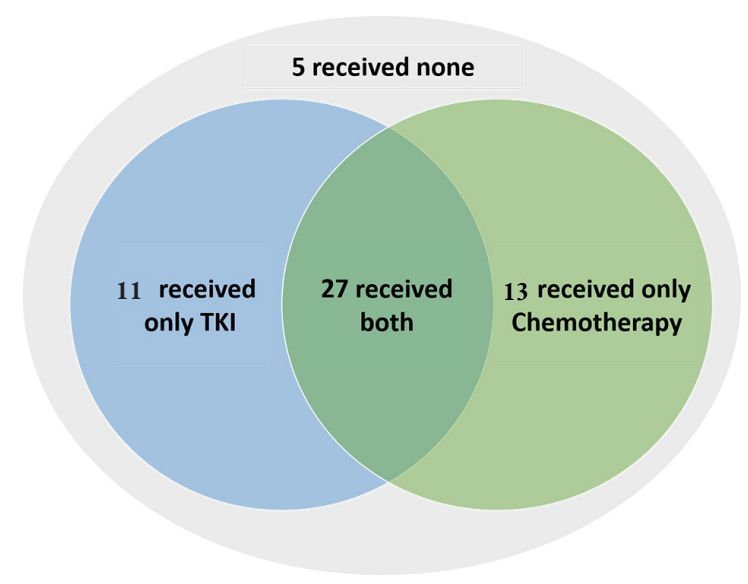

Figure S2 Distribution of patients according to treatment received.

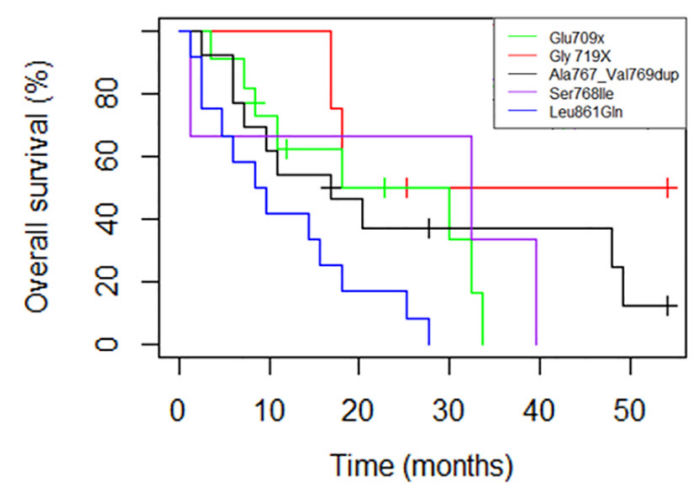

Figure S3 Median overall survival (mOS) for the 43 metastatic patients with single uncommon EGFR mutation, considering the EGFR uncommon mutation. p.Glu709X $(n=5): \operatorname{mOS}=18.0$ months [16.8-NA]; p.Gly719X) $(\mathrm{n}=20)$ : $\mathrm{mOS}=18.0$ months [10.8NA]; p.Ser768Ile $(\mathrm{n}=6)$ : $\mathrm{mOS}=32.4$ months [1.2-NA]; p.Ala767_ Val769dup (n=14): $\mathrm{mOS}=16.8$ months [7.2-NA]; p.Leu861Gln $(n=20):$ mOS $=9$ months [4.8-NA]. 
Table S2 Characteristics of metastatic patients harboring a single EGFR mutation

Total population $(n=45)$

\begin{tabular}{lc}
\hline Sex, $\mathrm{n}(\%)$ & $29(64.4)$ \\
Female & $16(35.6)$ \\
Male & $72.2(38.6-87.5)$ \\
Median age, years (range) & \\
Smoking history, $\mathrm{n}(\%)$ & $23(51.1)$ \\
Never & $19(42.2)$ \\
Current or former & $3(6.7)$ \\
Missing data & \\
Treatment, $\mathrm{n}$ (\%) & $21(46.7)$ \\
Targeted treatment and chemotherapy & $13(28.9)$ \\
Chemotherapy only & $9(20.0)$ \\
Targeted treatment only & $2(4.4)$ \\
None &
\end{tabular}
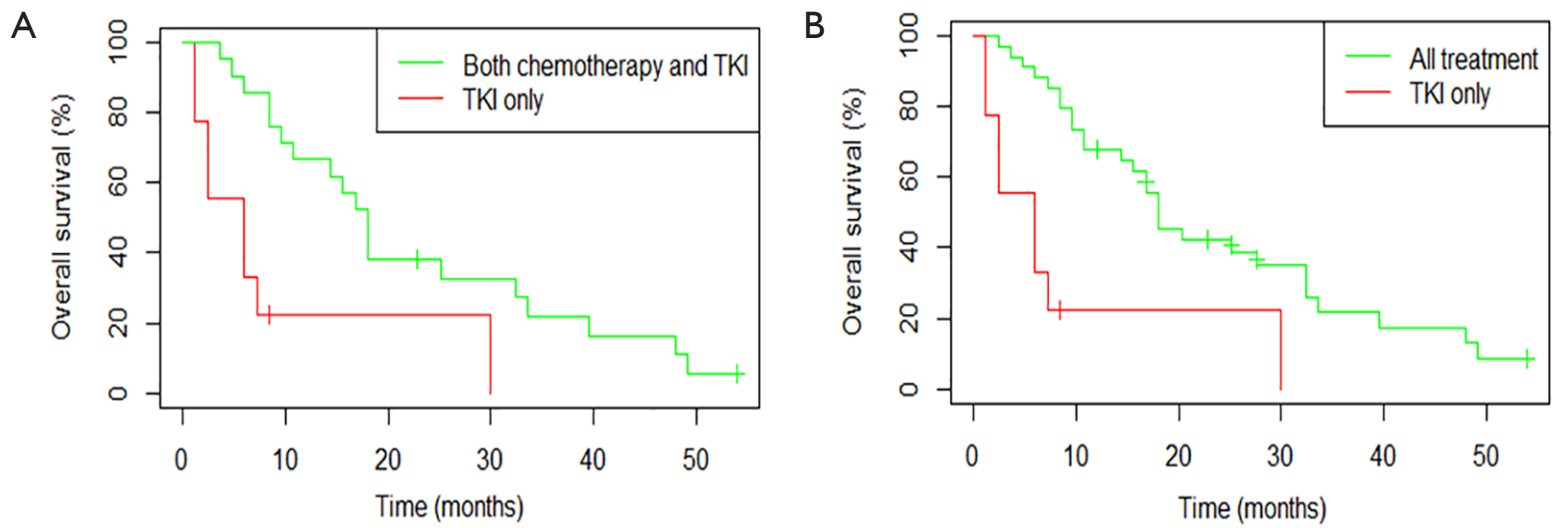

Figure S4 Overall survival (OS) for patients with a single uncommon EGFR mutation according to treatment, all lines. (A) Patients treated by TKI only $(n=9)$ were compared to patients treated by chemotherapy and TKI $(n=21)$. (B) Patients treated by TKI only ( $=9)$ were compared to patients treated by chemotherapy +/- TKI $(n=34)$. 
Table S3 Characteristics of metastatic patients harboring a double EGFR mutation

Total population $(n=11)$

$\begin{array}{lc}\text { Sex, } \mathrm{n}(\%) & 8(72.7) \\ \text { Female } & 3(27.3) \\ \text { Male } & 69.9(43.1-87.8) \\ \text { Median age, years (range) } & \\ \text { Smoking history, } \mathrm{n}(\%) & 7(63.6) \\ \text { Never } & 4(36.4) \\ \text { Current or former } & \\ \text { Treatment, n (\%) } & 6(54.5) \\ \text { Targeted treatment and chemotherapy } & 2^{\mathrm{b}}(18.2)^{\mathrm{a}} \\ \text { Targeted treatment only } & 0(0) \\ \text { Chemotherapy only } & 3(27.3)^{\mathrm{b}}\end{array}$

${ }^{a}$ including one patient with p.Leu858Arg co-mutation; ${ }^{\mathrm{b}}$ including two patients with p.Leu858Arg co-mutation. 
Exon 18 Glu709x

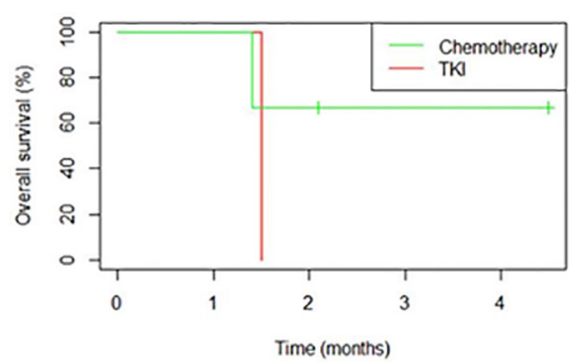

Exon 18 Gly $719 x$

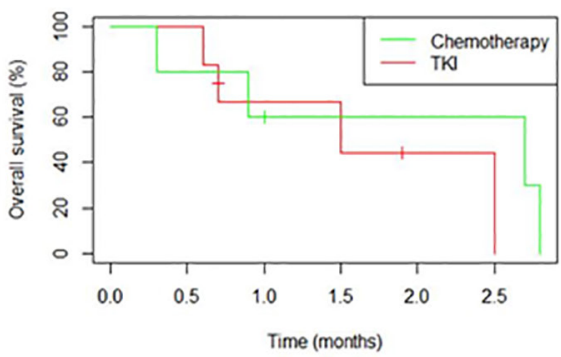

Exon 20 Ala767_Val769dup

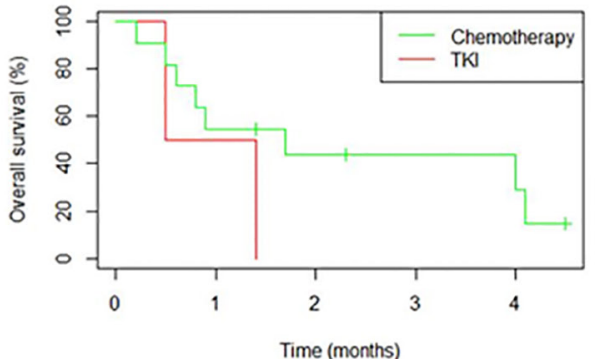

Exon 20 Ser768Ile

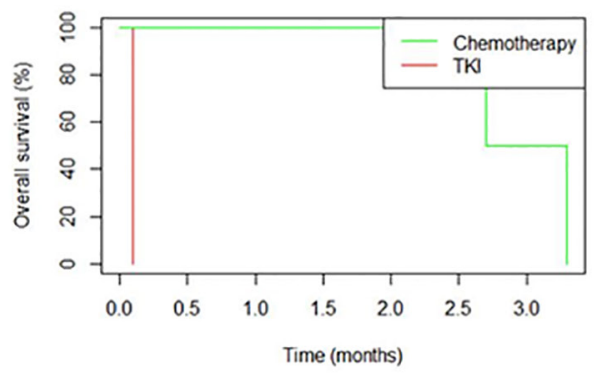

Exon 21 Leu861Gln

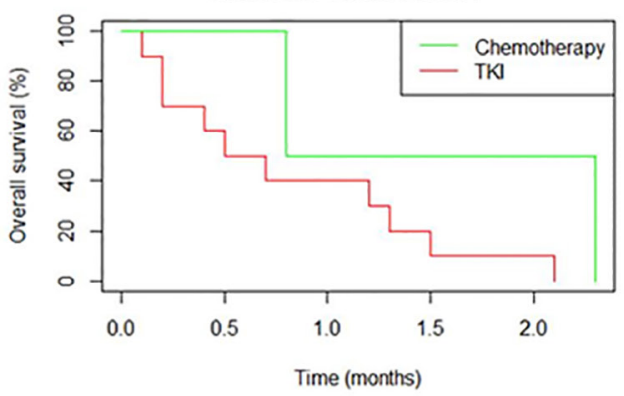

\begin{tabular}{|c|c|c|c|c|c|c|c|c|}
\hline $\begin{array}{l}\text { Single uncommon } \\
\text { EGFR mutation }\end{array}$ & $1^{\text {st }}$ line & $\mathbf{n}$ & mos & IC95\% & $\mathbf{P}$ & $\mathrm{HR}$ & IC95\% & $\mathbf{p}$ \\
\hline \multirow[t]{2}{*}{ Exon 18 Glu709x } & TKI & 1 & 1.5 & NA-NA & 0.5 & 0.41 & $0.25-6.62$ & 0.5 \\
\hline & $\begin{array}{l}\text { Chemotherap } \\
\text { y }\end{array}$ & 3 & NA & 1.4-NA & & & & \\
\hline \multirow[t]{2}{*}{ Exon 18 Gly719x } & TKI & 6 & 1.5 & $0.7-\mathrm{NA}$ & 0.4 & 0.51 & $0.09-2.87$ & 0.4 \\
\hline & $\begin{array}{l}\text { Chemotherap } \\
\text { y }\end{array}$ & 5 & 2.7 & $0.9-\mathrm{NA}$ & & & & \\
\hline \multirow[t]{2}{*}{$\begin{array}{l}\text { Exon } 20 \\
\text { Ala767_Val769du } \\
\text { p }\end{array}$} & TKI & 2 & 0.95 & $0.5-\mathrm{NA}$ & 0.2 & 0.4 & $0.08-2.1$ & 0.3 \\
\hline & $\begin{array}{l}\text { Chemotherap } \\
\text { y }\end{array}$ & 11 & 1.7 & $0.8-\mathrm{NA}$ & & & & \\
\hline \multirow[t]{2}{*}{$\begin{array}{l}\text { Exon } 20 \\
\text { Ser } 796811 \text { e } \\
\end{array}$} & TKI & 1 & 0.1 & NA-NA & 0.2 & $2.4 e^{\wedge}-10$ & 0 -inf & 0.2 \\
\hline & $\begin{array}{l}\text { Chemotherap } \\
\text { y }\end{array}$ & 2 & 3 & 2.7-NA & & & & \\
\hline \multirow[t]{2}{*}{$\begin{array}{l}\text { Exon } 21 \\
\text { Leu861GIn } \\
\end{array}$} & TKI & 10 & 0.6 & $0.2-\mathrm{NA}$ & 0.2 & 0.27 & $0.03-2.13$ & 0.2 \\
\hline & $\begin{array}{l}\text { Chemotherap } \\
\text { y }\end{array}$ & 2 & 1.55 & $0.8-\mathrm{NA}$ & & & & \\
\hline
\end{tabular}

Figure S5 Overall survival (OS) for the metastatic patients with single uncommon EGFR mutation $(\mathrm{n}=43)$ according to first-line treatment received and type of uncommon EGFR mutation. 